\title{
Beiträge zur Kenntnis der Harnsäurebildung.
}

\section{Mitteilung.}

Von

Dr. G. Izar, Assistenten des Instituts.

(Aus dem Institute für spezielle Pathologie innerer Krankheiten der K. Universität Pavia :

Professor M. Ascoli.)

(Der Redaktion zugegangen am 27. Januar 1910.)

In der 4. Mitteilung hat Preti1) nachgewiesen, daß die Wiederbildung zerstörter Harnsäure auf der Wirkung eines im Blute und im Blutserum enthaltenen Fermentes beruht.

Auf der anderen Seite steht die Tatsache, daß das (Hunde-) Serum ${ }^{1}$ ) allein die geringen, von ihm zerstörten Harnsäuremengen nicht wiederzubilden vermag, daß mithin ein oder mehrere andere Faktoren nötig sind.

Folgende Versuche $(1-3)$ zeigen, daß dieser unerläßliche Faktor in frischem oder gekochtem Leber- oder Milzbrei enthalten ist.

\section{Versuch I.}

$500 \mathrm{ccm}$ defibriniertes Rinderblut $+110,85 \%$ ige Chlornatriumlösung $+1,958 \mathrm{~g}$ Harnsäure $;^{2}$ ) nach 3 tägiger Autolyse, ${ }^{3}$ ) unter Luftdurchleitung, in 3 gleiche Portionen geteilt:

1) Diese Zeitschrift, Bd. LXII, S. 354 .

2) Der Zusatz erfolgte folgendermaßen: ca. $1 \mathrm{~g}$ (oder mehr) harnsaures Natron wurde in $100 \mathrm{ccm}$ (oder entsprechend mehr) Lithiumcarbonatlösung (1:90) gelöst. Auffüllung auf $200 \mathrm{ccm}$ mit destilliertem Wasser, Filtration; $175 \mathrm{ccm}$ (oder entsprechend mehr) der Lösung wurden zur Kolatur hinzugesetzt; in weiteren $25 \mathrm{ccm}$ wurde der Harnsäuregehalt bestimmt.

s) $\mathrm{Zu}$ den Flüssigkeiten wurde immer $2 \%$ Toluol und Chloroform hinzugesetzt und der Zusatz nach Unterbrechung der Luftdurchleitung in den weiter bei Abschluß von Luft im Brutschrank aufbewahrten Proben erneuert. 
A. Srofort koaguliert . . . . . . ergibt Harnsäure 254,48 mg.

B. Unter Zusatz von $50 \mathrm{ccm}$ Chlornatriumlössung: ${ }^{1}$ ) koaguliert nach weiterer 3 tägiger Autolyse im verschlossenen Gefäße. .

C. Unter Zusatz von $50 \mathrm{~g}$ gekochten Rinderleberbreies: koaguliert nach weiterer 3 tägiger Autolyse in verschlossenem Gefäße

\section{Versuch II.}

1,51 defibriniertes Rinderblut $+2,510,85 \%$ ige Chlornatriumlösung $\left.+\mathrm{ca.}^{2}\right) 3 \mathrm{~g}$ harnsaures Natron gelöst in Lithiumcarbonatlösung $(1: 90)$. Gesamtvolumen $4,5 \mathrm{l}$. Verteilung in 2 Portionen:

A. 1 I Flüssigkeit, sofort koaguliert . . . . ergibt Harnsäure 485,15 mg.

B. 3,5 l Flüssigkeit, nach 3 tägiger Autolyse

unter Luftdurchleitung in 4 Teile geteilt:

$\mathrm{B}_{1} .500 \mathrm{ccm}$ Flüssigkeit, sofort koaguliert.

$\mathrm{B}_{2} .1000 \mathrm{ccm}$ Flüssigkeit unter Zusatz)

von $50 \mathrm{~g}$ gekochten Rindermilzbreis

$\mathrm{B}_{3} .1000 \mathrm{ccm}$ Flüssigkeit unter Zusatz von $50 \mathrm{~g}$ gekochten Rinderleberbreis

$B_{4} .1000 \mathrm{ccm}$ Flüssigkeit unter Zusatz von $50 \mathrm{ccm}$ Chlornatriumlösung ... . .

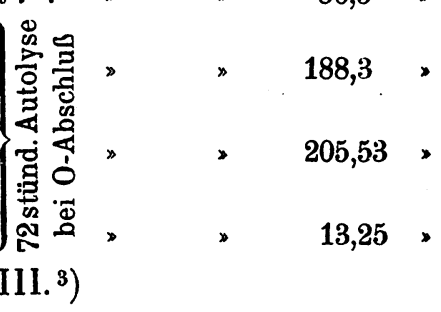

\section{Versuch III. ${ }^{3}$ )}

1,5 l defibriniertes Rinderblut $+2,510,85 \%$ ige Chlornatriumlösung. Verteilung in 2 Portionen (A, B):

A. 21 Flüssigkeit $+1,646 \mathrm{~g}$ Harnsäure in $250 \mathrm{ccm}$ Lithiumcarbonatlösung (1:90): 3 tägige Autolyse unter Luftdurchleitung, darauf weitere Verteilung in 4 gleiche Teile.

$\mathbf{A}_{1}$. Sofort koaguliert . . . . . . . ergibt Harnsäure 65,8 mg.

$A_{2}$. Unter Zusatz von $150 \mathrm{ccm}$ Chlornatriumlösung $\cdot \cdot \cdot \cdot \cdot \cdot \cdot \cdot \cdot \cdot \cdot \cdot$ natriumlösung und $50 \mathrm{~g}$ Rindermilzbrei

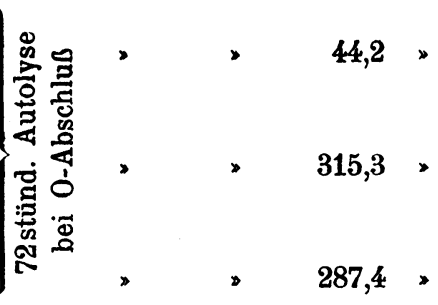

A. Unter Zusatz von $100 \mathrm{ccm}$ Chlornatriumlösung und $50 \mathrm{~g}$ gekochten Rindermilzbrei .........

1) Nach Unterbrechung der Luftdurchleitung wird immer in den weiter bei Abschluß von Luft im Brutschranke aufbewahrten Proben der Toluol- und Chloroformzusatz erneuert.

2) Nicht zur Gewichtskonstanz getrocknet.

s) Dieser Versuch bestätigt (vgl. Diese Zeitschrift, Bd. LVIII, S. 529), 
B. 21 Flüssigkeit $+250 \mathrm{ccm}$ Lithiumcarbonatlösung (1:90): 3 tägige Autolyse unter Luftdurchleitung, darauf weitere Verteilung 4 gleiche Teile: $B_{1}$. Wie $A_{1}$ behandelt ....... ergibt Harnsäure $0,0 \mathrm{mg}$. $B_{2} . A_{2}>$. 72 stünd. Autolyse $\Rightarrow \quad 0,0$ 》

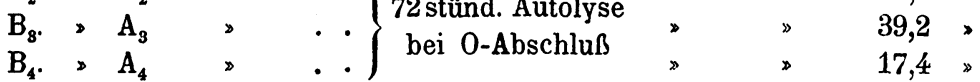

Der Zusatz geringer Mengen gekochter Leber genügt demnach, um die Wiederbildung der vom Blute zerstörten Harnsäure zu bewirken; größere Lebermengen. rufen weder Verminderung noch Vermehrung der wiedergebildeten Harnsäure hervor.

\section{Versuch IV.}

11 defibriniertes Rinderblut $+1,8510,85 \%$ ige Chlornatriumlösung + ca. 1,5 g harnsaures Natron gelöst in Lithiumcarbonatlösung (1:90). Gesamtvolumen $3 \mathrm{l}$. Verteilung in 2 Portionen (A, B):

A. $500 \mathrm{ccm}$ Flüssigkeit, sofort koaguliert . e ergibt Harnsäure $230,7 \mathrm{mg}$ -

B. 2,5 1 Flüssigkeit nach 3 tägiger Autolyse unter Luftdurchleitung in 5 Teile geteilt:

$\mathrm{B}_{\mathbf{1}} .500 \mathrm{ccm}$ Flüssigkeit, sofort koaguliert . .

$B_{2} .500 \mathrm{ccm}$ Flüssigkeit unter Zusatz von $250 \mathrm{ccm}$ Chlornatriumlösung ...

$\mathrm{B}_{3} .500 \mathrm{ccm}$ Flüssigkeit unter Zusatz von $240 \mathrm{ccm}$ Chlornatriumlösung und $10 \mathrm{~g}$ Rinderleberbrei ........

$B_{4} .500 \mathrm{ccm}$ Flüssigkeit unter Zusatz von $220 \mathrm{ccm}$ Chlornatriumlösung und $30 \mathrm{~g}$ Rinderleberbrei .......

$\mathrm{B}_{5} .500 \mathrm{ccm}$ Flüssigkeit unter Zusatz von $200 \mathrm{ccm}$ Chlornatriumlösung und $50 \mathrm{~g}$ Rinderleberbrei .......

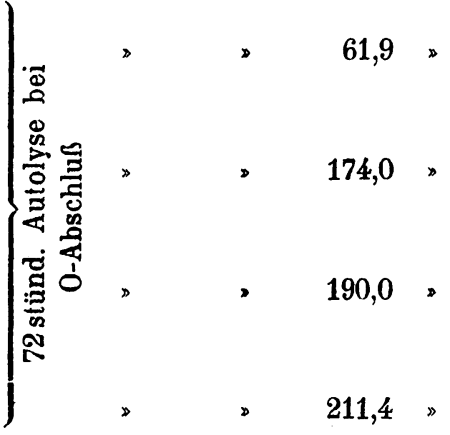

Aus Versuchen 1, 2, 3 geht hervor, dab das Leberoder Milzkoferment (Aktivator) thermostabil und alkohollöslich (Vers. 5-7) ist.

daß die Harnsäurewiederbildung nicht auf Kosten von in der Leber enthaltenen Substanzen erfolgt. 


\section{Versuch V.}

$700 \mathrm{ccm}$ defibriniertes Rinderblut $+1,610,85 \%$ ige Chlornatriumlösung + ca. $2 \mathrm{~g}$ harnsaures Natron in $200 \mathrm{ccm}$ Lithiumcarbonatlösung (1:90). 3tägige Autolyse im Brutschrank bei $53^{01}$ ) unter Luftdurchleitung, darauf Verteilung in 5 gleiche Teile :
A. Sofort koaguliert . . . . . . . . ergibt Harnsäure 75,75 mg.
B. + $100 \mathrm{ccm}$ Chlornatriumlösung -
C. + Rückstand des Alkoholextrak -
tes ${ }^{2}$ ) von $100 \mathrm{~g}$ Rinderleberbrei, in $100 \mathrm{ccm}$ Chlornatriumlösung aufge- schwemmt . . . . . . . .
D. + Ätherextrakt $\left.{ }^{2}\right)$ aus $100 \mathrm{~g}$ Rinder- leberbrei in $100 \mathrm{ccm}$ Chlornatrium- lösung aufgeschwemmt.....
E. + in Äther unlösliches, in Alkohol lösliches Extrakt ${ }^{2}$ ) aus $100 \mathrm{~g}$ Rinder- leberbrei, in $100 \mathrm{ccm}$ Chlornatrium- lösung aufgeschwemmt......

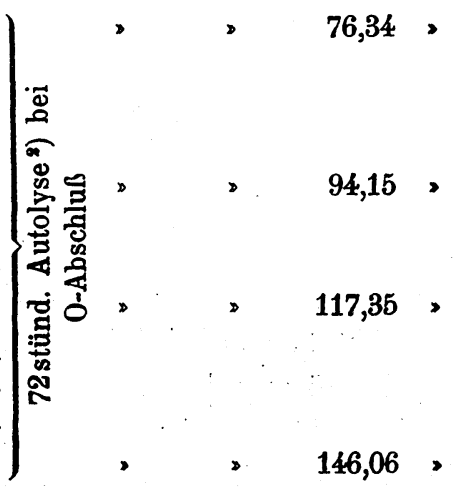

\section{Versuch VI.}

$400 \mathrm{ccm}$ defibriniertes Hundeblut $+800 \mathrm{ccm} 0,85 \%$ ige Chlornatriumlösung + ca. 2,5 g harnsaures Natron in $300 \mathrm{ccm}$ Lithiumcarbonatlösung (1:90). Gesamtvolumen 1,5 l. Verteilung in 2 Portionen (A, B):

A. $250 \mathrm{ccm}$ Flüssigkeit, sofort koaguliert . . ergibt Harnsäure $321,3 \mathrm{mg}$.

B. $1250 \mathrm{ccm}$ Flüssigkeit nach 3 tăgiger Autolyse im Brutschrank bei $53^{\circ}$ unter Luftdurchleitung in 5 gleiche Teile geteilt:

1) Hier und in Vers. VI u. VII blieb der Chloroform- und Toluolzusatz aus; die Fäulnis wurde durch Erhöhung der Brutschranktemperatur auf $53^{\circ}$ hintangehalten (L. Bellazzi, Diese Zeitschrift, Bd. LVII, S. 389).

2) Nach 0. Gohnheim (Diese Zeitschrift, Bd. XXXIX, S. 403).

Verschieden ist das Resultat, wenn der Auszug mit \& Volumina $95 \%$ igen Alkohols gefällt wird, anstatt denselben vorher nach Cohnheim einzudampfen. Im ersteren Falle (vgl. Vers. V und VI) wird das Koferment zum größten Teile im Niederschlage zurückgehalten und ist auch durch wiederholtes Waschen nur unvollständig zu entfernen.

Hoppe-Seyler's Zeitschrift f. physiol. Chemie. LXV. 
G. Izar,

$\mathrm{B}_{1}$. Sofort koaguliert . . . . . . . . ergibt Harnsäure 108,4 mg.

$\mathrm{B}_{2}+125 \mathrm{ccm}$ Chlornatriumlösung . . $\mathrm{B}_{3}+25 \mathrm{~g}$ gekochten Rinderleberbreis in $100 \mathrm{ccm}$ Chlornatriumlösung aufgeschwemmt. . . . . . . . . $\mathrm{B}_{4} \cdot+$ Alkoholniederschlag ${ }^{1}$ ) von $25 \mathrm{~g}$ Rinderleberbrei in $100 \mathrm{ccm}$ Chlornatriumlösung aufgeschwemmt . .

$\mathrm{B}_{5}$. + Alkoholextrakt ${ }^{1}$ ) aus $25 \mathrm{~g}$ Rinderleberbrei in $100 \mathrm{ccm}$ Chlornatriumlösung aufgeschwemmt . . . . .

X. $25 \mathrm{~g}$ gekochten Rinderleberbreis in $350 \mathrm{ccm}$ Chlornatriumlösung aufgeschwemmt . . . . . . . . .

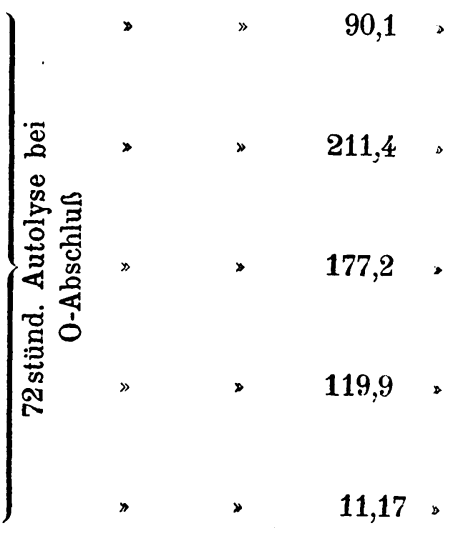

\section{Versuch VII.}

$500 \mathrm{ccm}$ defibriniertes Rinderblut $+1,410,85 \%$ ige Chlornatriumlösung + ca. $2 \mathrm{~g}$ harnsaures Natron in $200 \mathrm{ccm}$ Lithiumcarbonatlösung (1:90). 3 tägige Autolyse im Brutschrank bei $53^{\circ}$ unter Luftdurchleitung, darauf Verteilung in 7 gleiche Teile:

A. Sofort koaguliert . . . . . . ergibt Harnsäure 31,0 mg.

B. $+150 \mathrm{ccm}$ Chlornatriumlösung - .
C. $+50 \mathrm{~g}$ gekochten Rinderleberbreis in $100 \mathrm{ccm}$ Chlornatriumlösung aufgeschwemmt . . . . . . . .

D. + Rückstand nach Alkoholextraktion $^{2}$ ) von $50 \mathrm{~g}$ Rinderleberbrei in $100 \mathrm{ccm}$ Chlornatriumlösung aufgeschwemmt . . . . . . . . .

E. + Alkoholextrakt ") auf $50 \mathrm{~g}$ Rinderleberbrei in $100 \mathrm{ccm}$ Chlornatriumlösung aufgeschwemmt..... .

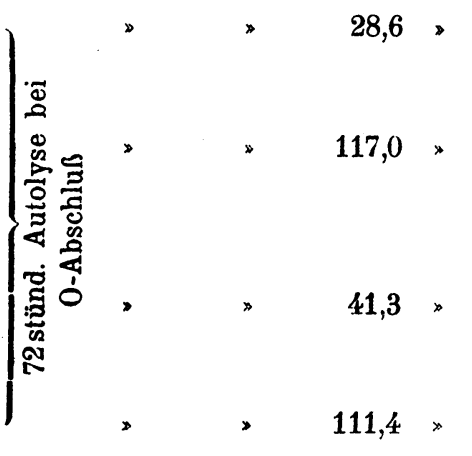

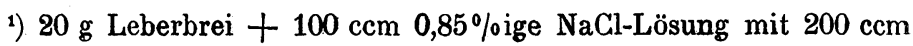
$95 \%$ igem Alkohol gefällt; Filtration. Der Niederschlag im Wasserbad bei $53^{\circ}$ zur Trockne eingedampft; desgleichen das Filtrat unter gleichzeitiger Aspiration im Vakuum. Die Rückstände getrennt in Kochsalzlösung aufgenommen.

2) Nach Ciohnheim (loc. cit.), jedoch ohne Ätherextraktion des Alkoholauszuges. 
F. + Alkoholniederschlag ${ }^{1}$ ) von $50 \mathrm{~g}$ ) Rinderleberbrei in $100 \mathrm{ccm}$ Chlornatriumlösung aufgeschwemmt . .

G. + Alkoholextrakt ') aus $50 \mathrm{~g}$ Rinderleberbrei in $100 \mathrm{ccm}$ Chlornatriumlösung aufgeschwemmt ..... ergibt Harnsäure 49,1 mg.

Früheren Versuchen ${ }^{2}$ ) nach besitzen Nierenauszüge nicht die Fähigkeit, die von ihnen bei Luftzufuhr zerstörte Harnsäure bei Luftabschluß wiederzubilden. Da nun die von uns benutzten Nieren immer mehr oder weniger Blut enthielten - es war uns nicht möglich, dieselben unmittelbar nach erfolgtem Schlachten der betreffenden Tiere $\mathrm{zu}$ bekommen und $\mathrm{zu}$ waschen - erschien es schon aus diesem Umstande nicht wahrscheinlich, daß das Koferment auch in der Niere enthalten sei. Tatsächlich ergibt sich aus Versuch 8 , daß Zusatz von Blut zu Nierenauszügen, die Harnsäure zerstört haben, nicht das Wiederauftreten derselben hervorruft.

Versuch VIII.

Kolatur aus $170 \mathrm{~g}$ Rindernierenbrei $+1,510,85 \%$ ige Chlornatriumlösung; Zusatz von 2,465 g Harnsäure in Lithiumcarbonatlösung (1:90). 3 tägige Autolyse unter Luftdurchleitung, darauf Verteilung in $\mathbf{5}$ gleiche Portionen:

A. Sofort koaguliert . . . . . . . ergibt Harnsäure 13,5 mg.

B. Unter Zusatz von $300 \mathrm{ccm}$ Chlornatriumlösung . . . . . . .

C. Unter Zusatz von $300 \mathrm{ccm}$ defibriniertem Rinderblut. . . . . . .

D. Gekocht, darauf wie B behandelt

E.

X. $300 \mathrm{ccm}$ defibriniertes Rinderblut unter Zusatz von $500 \mathrm{ccm}$ Chlornatriumlösung .........

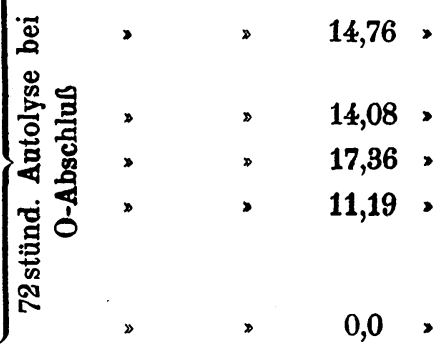

Hingegen erwarteten wir, daß der Zusatz von Koferment enthaltendem, gekochtem Leberextrakte Harnsäurewiederbildung

1) Technik wie in Versuch VI, der Alkoholniederschlag wieder mit Alkohol aufgenommen, geschüttelt, filtriert und die Prozedur ein zweites Mal wiederholt, die Filtrate vereinigt und wie gewöhnlich behandelt.

2) M. Ascoli und G. Izar, Diese Zeitschrift, Bd. LVIII, S. 538. 
seitens der Nieren hervorzurufen imstande wäre: gegen jede Erwartung fielen aber die dahin zielenden Experimente negativ aus (Vers. 9). Nur der Zusatz von frischem oder autolysiertem Milzbrei (Vers. 9, 10, 11, 12) oder Leberbrei, nicht aber von gekochtem, führt zur Harnsäurewiederbildung.

\section{Versuch IX.}

Kolatur aus $150 \mathrm{~g}$ Rindernierenbrei $+1,510,85 \%$ ige Chlornatriumlösung: Zusatz von ca. $2 \mathrm{~g}$ harnsaurem Natron in Lithiumcarbonatlösung $(1: 90)$. 3 tägige Autolyse unter Luftdurchleitung, darauf Verteilung in 4 gleiche Portionen:

1. Sofort koaguliert . . . . . . ergibt Harnsäure $74,3 \mathrm{mg}$.

2. Unter Zusatz von $100 \mathrm{ccm}$ Chlor-) natriumlösung ........

3. Unter Zusatz von $20 \mathrm{~g}$ gekochten Rinderleberbreis und $80 \mathrm{ccm}$ Chlornatriumlösung ........

4. Unter Zusatz von $20 \mathrm{~g}$ frischen Rinderleberbreis und $80 \mathrm{ccm}$ Chlornatriumlösung

a) $20 \mathrm{~g}$ gekochten Rinderleberbreis in $600 \mathrm{ccm}$ Chlornatriumlösung ... .

b) $20 \mathrm{~g}$ frischen Rinderleberbreis in $600 \mathrm{ccm}$ Chlornatriumlösung ....

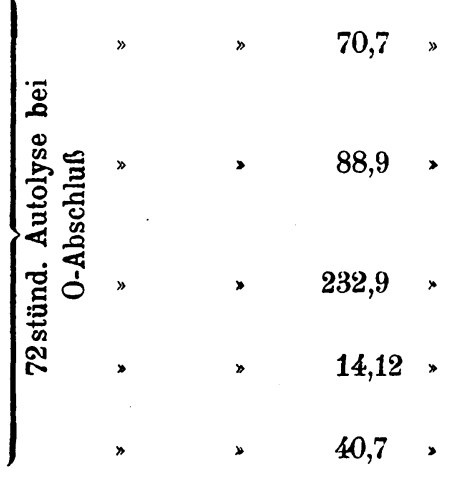

\section{Versuch X.}

Kolatur aus $200 \mathrm{~g}$ Rindernierenbrei $+1,610,85^{\circ}$ ioige Chlornatriumlösung: Zusatz von ca. $5 \mathrm{~g}$ harnsaurem Natron in Lithiumcarbonatlösung $(1: 90)$. 3tägige Autolyse unter Luftdurchleitung, darauf Verteilung in 4 gleiche Portionen:

a) Sofort koaguliert . . . . . . . ergibt Harnsäure 22,39 mg.

b) Unter Zusatz von $750 \mathrm{ccm}$ Chlor-) natriumlösung ........

c) Unter Zusatz von $750 \mathrm{ccm}$ autolysierter Kolatur aus Rindermilzbrei $\left(\alpha_{1}\right)$..

d) Unter Zusatz von $750 \mathrm{ccm}$ frischer Kolatur aus Rindermilzbrei $\left(\beta_{1}\right) \ldots$.

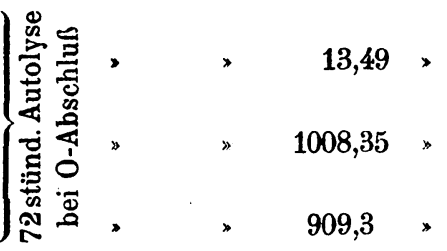


$\alpha=1,5$ l Kolatur aus $140 \mathrm{~g}$ Rindermilzbrei $+1,4$ l Chlornatriumlösung: 3 tägige Autolyse unter Luftdurchleitung, darauf Verteilung in 2 gleiche Portionen $\left(\alpha_{1}-\alpha_{2}\right)$ :

$\alpha_{1}=750 \mathrm{ccm}$ Kolatur: $z u$ c) hinzugesetzt.

$\alpha_{2}=750 \mathrm{ccm}$ Kolatur: 72 stünd. Autolyse

bei 0 -Abschluß . . . . . . . . ergibt Harnsäure 101,61 mg.

$\beta=1,51$ Kolatur aus $140 \mathrm{~g}$ Rindermilzbrei $+1,41$ Chlornatriumlösung. Verteilung in 2 gleiche Portionen $\left(\beta_{1}-\beta_{2}\right)$ :

$\beta_{1}=750 \mathrm{ccm}$ Kolatur: $\mathrm{zu}$ d) hinzugesetzt.

$\beta_{2}=750 \mathrm{ccm}$ Kolatur: 72 stünd. Autolyse

bei O-Abschluß. . . . . . . ergibt Harnsäure $86,9 \mathrm{mg}$.

\section{Versuch XI.}

A. Kolatur aus $150 \mathrm{~g}$ Rindernierenbrei $+1,510,85 \%$ ige Chlornatriumlösung: Zusatz von 2,353 g Harnsäure in Lithiumcarbonatlösung (1:90). 3 tägige Autolyse unter Luftdurchleitung, darauf Verteilung in 7 gleiche Portionen:

1. Sofort koaguliert . . . . . . ergibt Harnsäure 68,1 mg.

2. Unter Zusatz von $400 \mathrm{ccm}$ Chlornatriumlösung ............

3. Gekocht: unter Zusatz von $400 \mathrm{ccm}$ Chlornatriumlösung . . . . . .

4. Gekocht: unter Zusatz von $400 \mathrm{ccm}$ autolysierter Kolatur aus Rindermilzbrei (b) . . . . . . . .

5. Gekocht: unter Zusatz von $400 \mathrm{ccm}$ autolysierter und gekochter Kolatur aus Rindermilzbrei (d) . . . .

6. Gekocht: unter Zusatz von $400 \mathrm{ccm}$ frischer Kolatur aus Rindermilzbrei ( $\beta$ )

7. Gekocht: unter Zusatz von $400 \mathrm{ccm}$ frischer,gekochter Kolatur aus Rindermilzbrei $(\delta) \ldots \ldots \ldots . .$.

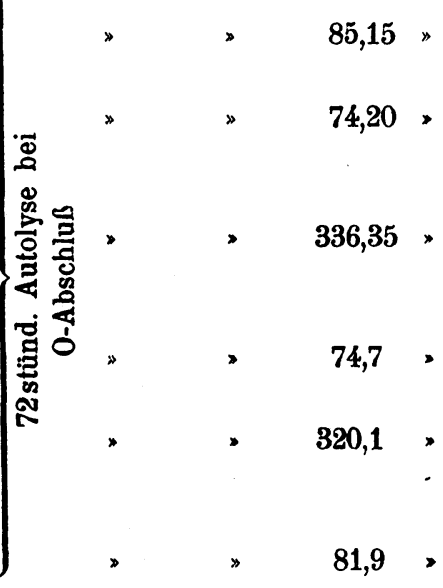

B. Kolatur aus 200.g Rindermilzbrei +21 Chlornatriumlösung. 3 tägige Autolyse unter Luftdurchleitung, darauf Verteilung in 4 gleiche Portionen:

a) $400 \mathrm{ccm}$ Kolatur: 72 stünd. Autolyse bei 0 -Abschluß......... ergibt Harnsäure 65,0 mg.

b) $400 \mathrm{ccm}$ Kolatur: zu 4. hinzugesetzt. 
c) $400 \mathrm{ccm}$ Kolatur: gekocht, darauf wie a)

behandelt . . . . . . . . ergibt Harnsäur 7,9 mg.

d) $400 \mathrm{ccm}$ Kolatur: gekocht, zu 5. hinzugesetzt.

C. Kolatur aus 200 g Rindermilzbrei + 21 Chlornatriumlösung. Verteilung in 4 gleiche Portionen:

a) $400 \mathrm{ccm}$ Kolatur: 72 stünd. Autolyse bei 0-Abschluß. . . . . . . ergibt Harnsäure 40,2 mg.

ß) $400 \mathrm{ccm}$ Kolatur: zu 6. hinzugesetzt.

r) $400 \mathrm{ccm}$ Kolatur: gekocht, darauf wie $\alpha)$ behandelt .......... \ $\ldots 10,7$,

d) $400 \mathrm{ccm}$ Kolatur: gekocht, zu 7. hinzugesetzt.

Versuch XII.

Kolatur aus $170 \mathrm{~g}$ Rindernierenbrei $+1,7$ l 0,85\% ige Chlornatriumlösung: Zusatz von ca. $3 \mathrm{~g}$ harnsaurem Natron in Lithiumcarbonatlösung $(1: 90)$.

3 tägige Autolyse unter Luftdurchleitung, darauf Verteilung in 7 gleiche Portionen:

1. Sofort koaguliert . . . . . . . . ergibt Harnsäure $24,2 \mathrm{mg}$

2. Unter Zusatz von $200 \mathrm{ccm}$ Chlornatriumlösung . . . . . . .

3. Unter Zusatz von $200 \mathrm{ccm}$ Kolatur aus Rindermilzbrei .......

4. Unter Zusatz von $200 \mathrm{ccm}$ gekochter Kolatur aus Rindermilzbrei ....

5. Gekocht: unter Zusatz von $200 \mathrm{ccm}$ Chlornatriumlösung . . . . . .

6. Gekocht: unter Zusatz von $200 \mathrm{ccm}$ Kolatur aus Rindermilzbrei .... .

7. Gekocht: unter Zusatz von $200 \mathrm{ccm}$ gekochter Kolatur aus Rindermilzbrei

\begin{tabular}{|c|c|c|c|}
\hline \multirow{5}{*}{ 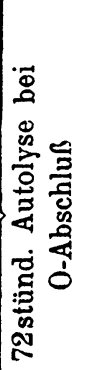 } & $\triangleright$ & 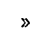 & 29,7 \\
\hline & > & 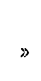 & 214,0 \\
\hline & $D$ & 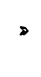 & 41,0 . \\
\hline & 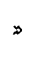 & , & 31,2 , \\
\hline & $\triangleright$ & , & 238,0 \\
\hline & & $»$ & \\
\hline
\end{tabular}

Kolatur aus $120 \mathrm{~g}$ Rindermilzbrei $+1,21$ Chlornatriumlösung. Verteilung in 6 gleiche Portionen:

a) $200 \mathrm{ccm}$ Kolatur . . . 72 72 stünd. Autolyse ergibtHarnsäure 44,2 $\mathrm{mg}$

b) 200 gekochte Kolatur $\}$ bei 0-Abschluß , > 11,7 ,

c) 200 Kolatur: Zu 3. hinzugesetzt

d) $200 \triangleright \quad: \quad$ \& 6 
e) $200 \mathrm{ccm}$ gekochte Kolatur: $\mathrm{Zu}$ 4. hinzugesetzt

f) 200 " : $>7$. "

Daraus ergab sich die Schlußfolgerung, daß beide die Wiederbildung bewerkstelligenden Faktoren in der Niere fehlen oder nur in minimalen Mengen vorhanden sind. Möglich schien es immerhin, daß das Ausbleiben der Harnsäurewiederbildung von der Anwesenheit diesen Prozeß hemmender Körper abhinge; allein die zur Prüfung dieser Möglichkeit vorgenommenen Experimente (Vers. 13-16) fielen nicht zugunsten dieser Vermutung aus; die Harnsäurewiederbildung vollzog sich, sobald den Nierenbreikolaturen, nach geleisteter Harnsäurezerstörung, frisches defibriniertes Blut und gekochter Leber- oder Milzbrei zugesetzt wurden. $\left.{ }^{1}\right)$

\section{Versuch XIII.}

Kolatur aus $130 \mathrm{~g}$ Rindernierenbrei $+1,310,85 \%$ ige Chlornatriumlösung: Zusatz von 2,6515 g Harnsäure in Lithiumcarbonatlösung $(1: 90)$. 3 tägige Autolyse unter Luftdurchleitung; darauf Verteilung in 5 gleiche Portionen:

1. Sofort koaguliert . . . . . . . ergibt Harnsäure $26,8 \mathrm{mg}$

2. Gekocht: unter Zusatz von $200 \mathrm{ccm}$ Chlornatriumlösung . . . . . .

3. Gekocht: unter Zusatz von $100 \mathrm{ccm}$ defibriniertem Rinderblut und $100 \mathrm{ccm}$ Chlornatriumlösung . . . . . .

4. Gekocht: unter Zusatz von $100 \mathrm{ccm}$ defibriniertem Rinderblut und $20 \mathrm{~g}$ Rindermilzbrei und $80 \mathrm{ccm}$ Chlornatriumlösung ........

5. Gekocht: unter Zusatz von $100 \mathrm{ccm}$ defibriniertem Rinderblut und $20 \mathrm{~g}$ Rinderleberbrei und $80 \mathrm{ccm}$ Chlornatriumlösung .........

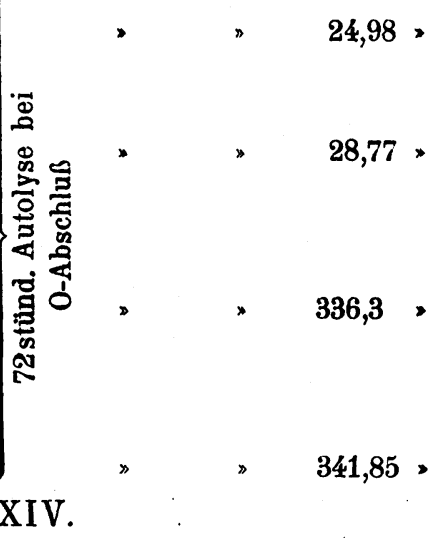

\section{Versuch XIV.}

Kolatur auf $160 \mathrm{~g}$ Rindernierenbrei $+1,610,85 \%$ ige

1) Weiter war es noch denkbar, daß ein Überwiegen der Uricolyse vorliege; gegen diese Annahme spricht aber Versuch 8, demgemäß auch beim Kochen der Nierenextrakte vor dem Blutzusatze Harnsäurewiederbildung nicht zutage tritt. 
Chlornatriumlösung: Zusatz von 2,693 g Harnsäure in Lithiumcarbonatlösung $(1: 90)$.

3tägige Autolyse unter Luftdurchleitung, darauf Verteilung: in 5 gleiche Portionen:

1. Sofort koaguliert . . . . . . . ergibt Harnsäure 134,4 ng;

2. Gekocht: unter Zusatz von $200 \mathrm{ccm}$ Chlornatriumlösung . . . . . .

3. Gekocht: unter Zusatz von $100 \mathrm{ccm}$ defibriniertem Rinderblut und $100 \mathrm{ccm}$ Chlornatriumlösung . . . . . .

4. Gekocht: unter Zusatz von $100 \mathrm{ccm}$ defibriniertem Rinderblut und $20 \mathrm{~g}$ Rindermilzbrei in $80^{\circ} \mathrm{ccm}$ Chlornatriumlösung aufgeschwemmt. . .

j. Gekocht: unter Zusatz von $100 \mathrm{ccm}$ defibriniertem Rinderblut und $20 \mathrm{~g}$ Rinderleberbrei in $80 \mathrm{ccm}$ Chlornatriumlösung aufgeschwemmt . . .

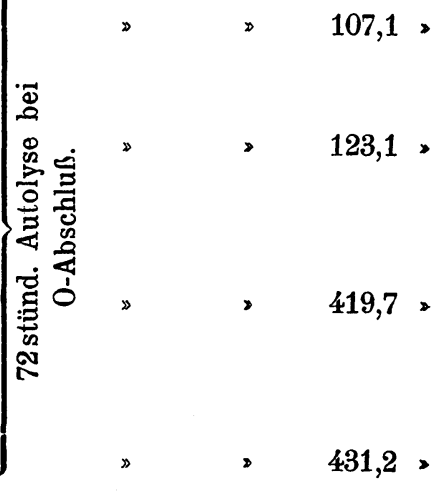

a) $100 \mathrm{ccm}$ defibriniertes Rinderblut unter Zusatz von $100 \mathrm{ccm}$ Chlornatriumlösung . . . . . . .

b) $100 \mathrm{ccm}$ defibriniertes Rinderb/ut unter Zusatz von $20 \mathrm{~g}$ Rindermilzbrei in $80 \mathrm{ccm}$ Chlornatriumlösung aufgeschwemmt . . . . . .

c) $100 \mathrm{ccm}$ defibriniertes Rinderblut unter Zusatz von $20 \mathrm{~g}$ Rinderleberbrei in $80 \mathrm{ccm}$ Chlornatriumlösung aufgeschwemmt . . . . . . .

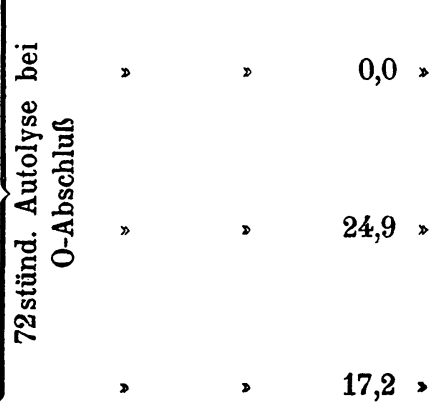

Selbstverständlich gelten unsere Angaben nur für Kalbsleber.

Schlußsatz.

Die Wiederbildung zerstörter Harnsäure beruht auf dem Zusammenwirken des schon nachgewiesenen im Blute enthaltenen Fermentes und eines in Leber und Milz, nicht aber in der Niere vorkommenden, koktostabilen und alkohollöslichen Kofermentes. 